\section{An algorithm to generate dynamic point-light displays}

\author{
KIT H. SHUM \\ University of California, Irvine, California 92717
}

The work of Johansson (1950) initiated some in teresting research on motion perception using dynamic pointlight displays (Barclay, Cutting, \& Kozlowski, 1978; Johansson, 1973, 1975, 1976; Proffitt \& Cutting, 1979; Proffitt, Cutting, \& Stier, 1979). The idea is to limit the scope of the essential motion stimulus to a manageable number of strategically placed point-lights in a blackout viewing condition. Such displays consist only of a number of light dots, each moving in a particular trajectory. But the richness of the information conveyed through such "degraded" representations can be fully appreciated only by watching the actual visual displays.

In most of such studies, the motion displays were obtained by recording the physically moving pointlights directly, either on film or on videotape. For example, Barclay et al. (1978) attached light-reflecting tape on a human body and video-recorded the person walking; Proffitt and associates mounted disks of reflecting tape on a rolling coffee can. On the other hand, given that the motion is lawful, it should be possible to simulate these displays using computer graphics. This has, in fact, been done, for example, for a walking figure (Cutting, 1978a, 1978b).

The basic problem is to specify the coordinates of the successive positions of individual points. For example, in the "rolling wheel" displays, a point-light mounted on the rim of the "wheel" can be specified by the following coordinate functions: $\mathrm{x}=\mathrm{r}[\mathrm{w} \cdots \sin (\mathrm{w})]$ and $y=r[1-\cos (w)]$, where $r$ is the radius of the "wheel," and $w$ is the angular displacement. In general, as long as these coordinate functions are defined, the successive positions of this point-light can be plotted on a screen sequentially to simulate the effect.

Shum (1980) has proposed that any cyclical motion can be approximated by a finite set of orthogonal sinusoidal components. This suggests a simple way of creating dynamic point-light displays through a recursive loop that sums up the sinusoids. For example, a circular motion can be simulated with two orthogonal components of equal amplitude and frequency, but differing by a phase of $90 \mathrm{deg}$. In other words, the coordinate functions are: $x=r \sin (w)$ and $y=r \cos (w)=r \sin (w+$ $90 \mathrm{deg})$. Ellipses of different eccentricities can be simulated by varying the ratio between the amplitudes; that is $x=a \sin (w)$ and $y=b \cos (w)$. A cycloid (the rolling wheel) can be created by adding a horizontal low-frequency but high-amplitude component (to approximate the linear translation) to the basic circular motion. Thus, $x=r \sin (w)+k r \sin (w / k)$ and $y=r \cos (w)$, where $k$ is a large integer. This Fourier-synthetic technique is rather powerful. For example, a pendular motion can be simulated with two components of different frequencies, and a square path, by 10 orthogonal components, with satisfactory results.

The author has implemented a display system on a Terak 8510/a microcomputer, based on this idea of Fourier synthesis. The program is written in UCSD PASCAL. But the basic algorithm can be easily demonstrated with a simple BASIC program, which is shown in the appendix. The core of this program lies in the loops from Statements 320 to 380 , which sum up the sinusoidal components for the $\mathrm{x}$ and $\mathrm{y}$ coordinates. The Terak has a 240 by 320 dot matrix screen. Light dots can be plotted and erased (before plotting the next position) with a graphics intrinsic called DRAWBLOCK. Satisfactory motion displays can be generated for up to five distinct dots in a display.

Requests for additional information can be addressed to the author, care of School of Social Sciences, University of California, Irvine, California 92717.

\section{REFERENCES}

Barclay, C. B., Cutting, J. E., \& Kozlowski, L. T. Temporal and spatial factors in gait perception that influence gender recognition. Perception \& Psychophysics, 1978, 23, 145-152.

CutTing, J. E. Generation of synthetic male and female walkers through manipulation of a biomechanical invariant. Perception, $1978,7,393-405$. (a)

Cutring, J. E. A program to generate synthetic walkers as dynamic point-light displays. Behavior Research Methods \& Instrumentation, 1978, 10, 91-94. (b)

Johansson, G. Configuration in event perception. Uppsala, Sweden: Almqvist \& Wiksell, 1950.

JoHAnsson, G. Visual perception of biological motion and a model for its analysis. Perception \& Psychophysics, 1973, 14, 201-211.

Joн Ansson, G. Visual motion perception. Scientific American, 1975, 232(6), 76-89.

JoHANSSON, G. Spatio-temporal differentiation and integration in visual motion perception. Psychological Research, 1976, 38, 379-393.

Proffitt, D. R., \& Cutting, J. E. Perceiving the centroid of configurations on a rolling wheel. Perception \& Psychophysics, 1979, 25, 389-398.

Proffitt, D. R., \& Cutting, J. E., \& Stier, D. M. Perception of wheel-generated motions. Journal of Experimental Psychology: Human Perception and Performance, 1979, 5, 289-302.

Shum, K. H. A quantitative study of perceptual vector analysis. Unpublished doctoral dissertation, Dartmouth College, 1980. 
Appendix

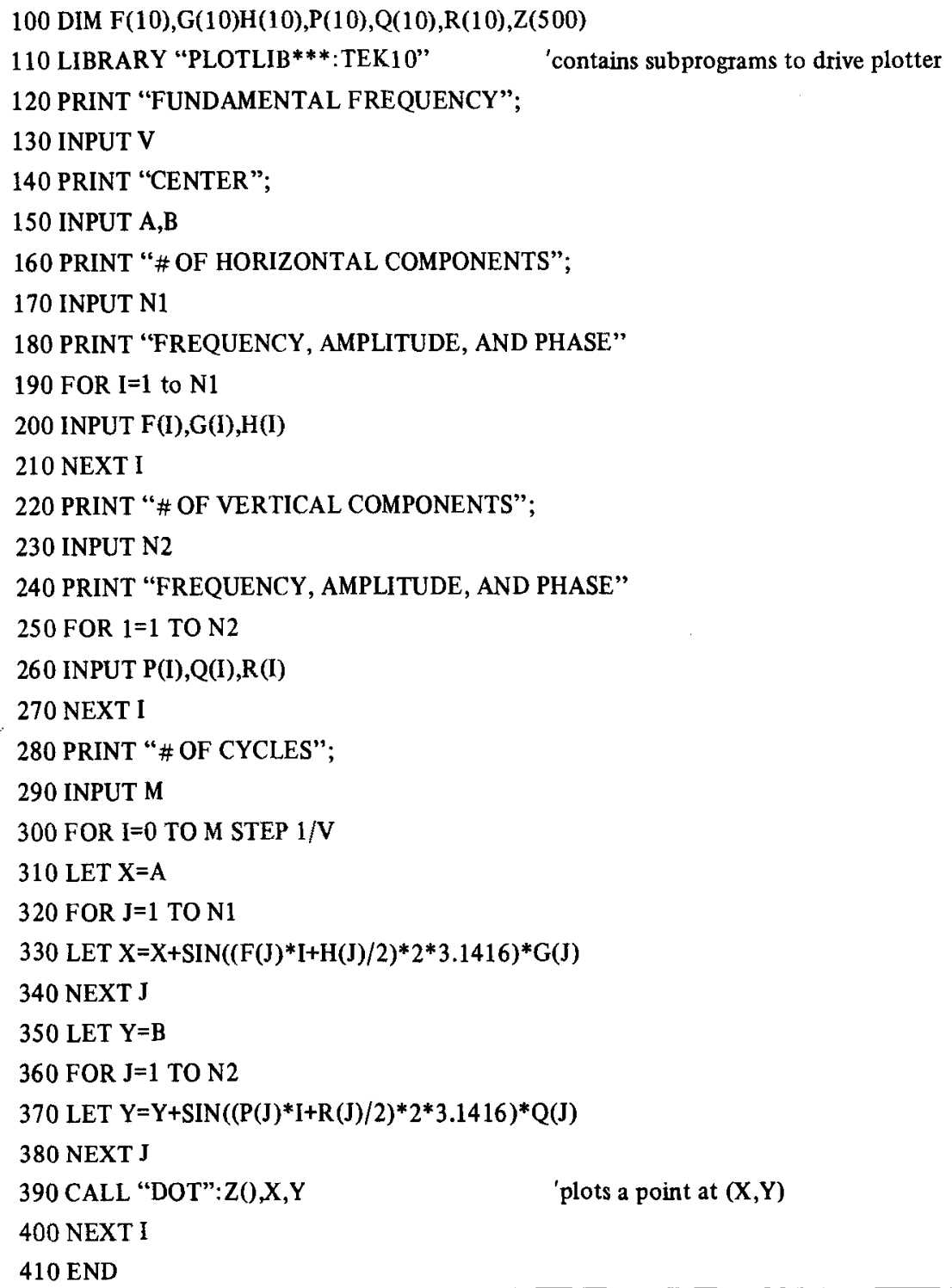

